of protected areas varies around the world, and biodiversity scientists have long suspected that areas with better safeguards to stop illegal encroachment for logging, agriculture or other activities are more likely to be a safe haven for biodiversity. However, demonstrating that such exclusion is effective is not straightforward.

Assessments of ecosystems and biodiversity are hampered by piecemeal data collection that uses incomparable methodologies, and the data have large gaps. That makes it difficult to draw broad conclusions about the global health of biodiversity, which many scientists say is a key reason why biodiversity has failed to climb political agendas, even as extinctions of animals and plants continue to rise. Around one-fifth of all 5,490 described mammal species are at risk of extinction, according to data from the International Union for Conservation of Nature.

These difficulties are driven by funding constraints and the intricacies of monitoring and assessing complex ecosystems. Nevertheless, some informative evaluations do exist, including the biennial Living Planet Index drawn up by the conservation group WWF, which follows trends in populations of species around the world to give indications of the state of global biological diversity.

More of these data gaps have now been filled, thanks to an assessment of protected tropical forest areas led by William Laurance, a conservation biologist at James Cook University in Cairns, Australia, which offers a snapshot of global biodiversity and reviews the success of this tool.

In a Herculean effort, Laurance and his team, along with more than 100 co-authors, systematically collected standardized data on environmental changes over the past $20-30$ years in 60 protected areas across the world's major tropical regions of Africa, America and Asia. The data include changes in the abundance of 31 groups of species, including primates, freshwater fish and exotic plants, and 21 potential

drivers of environmental change, such as road building and hunting.

To gather the information, the team conducted 262 interviews with field biologists and environmental scientists, and asked them each to complete a detailed 10-page questionnaire. It took the team around four years to gather the data, but Laurance reckons that to do so from scratch would cost billions of dollars and take $20-30$ years. The effort paid off, and the results appear online on Nature's website this week

"Around half of the reserves are experiencing a severe loss of biodiversity."
(W. Laurance et al. Nature http://dx.doi. org/10.1038/nature11318; 2012).

The team found that around half of the reserves are experiencing a severe loss of biodiversity. Crucially, the researchers also demonstrated the negative impact that environmental changes immediately outside the areas have on the health of the habitat inside the reserves. And they found that destructive activities such as forest clearance, fires and logging increasingly reach up to the edge of the protected areas. The results show that $85 \%$ of the reserves experienced declines in surrounding forest cover over the study period, whereas only $2 \%$ gained forest. These findings will not surprise biodiversity scientists, who have long been aware of such trends, but now have the data to show it.

The work highlights, yet again, the growing challenges that threaten the success of the protected-reserves model. Many reserves simply do not function as intended, but there are few alternative approaches. The results underscore the importance of better management of areas around the reserves. Rather than treating these ecological havens as islands, Laurance and his team recommend implementing buffer zones around protected areas to cushion the blow. The reserves can protect wildlife, but we must first protect the reserves.

\section{Error prone}

\section{Biologists must realize the pitfalls of work on massive amounts of data.}

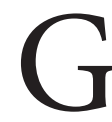
enomics has the potential to revolutionize medical care, but it is becoming increasingly clear that the field is having to deal with growing pains.

In a Comment piece this week, Daniel MacArthur, a researcher at Massachusetts General Hospital in Boston, argues that the massive pools of data generated in even routine genome studies make it easy to misinterpret artefacts as biologically important results (see page 427). Such false positives, he says, can lead to embarrassing retractions, futile projects and stalled careers. More careful attention to methods and greater awareness of the potential pitfalls will help to cut down on the needless mistakes.

In a field as competitive as genomics, scientists will inevitably seek faster, more efficient ways to generate and analyse data. Just this week, the firm Ion Torrent in Guilford, Connecticut - part of Life Technologies in Carlsbad, California - announced that it will tackle a competition to accurately sequence 100 genomes in 30 days for less than US $\$ 1,000$ per genome - and to win the US\$10-million prize offered by the X Prize Foundation in Playa Vista, California (see page 417).

Genomics is not the only field of science to battle with qualitycontrol issues. In March, Nature lamented the high number of corrections to research papers in the life sciences that arise from avoidable errors (see Nature 483, 509; 2012). Scientists are making too many careless mistakes, and those mistakes are getting published.

Much of this sloppy science comes from the pressure to generate 'surprising' results and to publish them quickly, even though they are more likely to be driven by errors than are findings that more or less follow from previous work. A researcher who reveals something

exciting is more likely to get a high-profile paper (and a permanent position) than is someone who spends years providing solid evidence for something that everyone in the field expected to be true.

This pressure extends throughout the careers of scientists, and is compounded by the preference of journals (including Nature) to publish significant findings - and of the media to report them. MacArthur asks scientists to weigh up the importance of avoiding being scooped against the embarrassment of a mistake, but to an ambitious scientist in a competitive field such as genomics, the risk of being out-published will often outweigh the potential damage of retraction.

Many areas of the life sciences now work with massive amounts of data, so technology-based artefacts are unlikely to be restricted to genomics. Any life scientist who works at a university or is affiliated with a hospital can now collect human samples and sequence them to create huge amounts of genomic data, with which they are perhaps not used to working. The problem goes beyond analysis - time and time again, biologists fail to design experiments properly, and so submit underpowered studies that have an insufficient sample size and trumpet chance observations as biological effects.

The problems are not hard to solve. Biologists must seek relevant training in experimental methods and collaborate with good statisticians. Principal investigators have a responsibility to their labs and to colleagues to ensure that any data they publish are robust. And the efforts of peer reviewers who thoroughly reanalyse data to doublecheck that submissions are solid deserve more formal acknowledgement, albeit in private.

Meanwhile, researchers who deal with large amounts of data must agree on standards that will protect against avoidable errors. Fields such as RNA sequencing have been slow to establish such guidelines (see Nature 484, 428; 2012), but others have shown that it can be done.

$\rightarrow$ NATURE.COM To comment online, click on Editorials at: go.nature.com/xhunqu
The human-genetics community, for instance, has established criteria for genome-wide association studies to ensure that findings are rigorous and comparable. Less-proactive genomics fields, and the rest of biology, should follow that lead. 